Classification

Physics Abstracts

78.20Bh $-78.65 \mathrm{Pi}-71.10+\mathrm{x}$

\title{
PSTM: An alternative to measure local variation of optical index
}

\author{
Dominique Barchiesi and Daniel Van Labeke \\ Laboratoire d'Optique P.M. Duffieux, URA CNRS 214, Université de Franche-Comté, Route de \\ Gray, 25030 Besançon Cedex, France
}

(Received July 4; accepted December 16, 1994)

\begin{abstract}
Résumé. - Les Microscopes Tunnel Optiques à balayage (STOM : Scanning Tunneling Optical Microscopes ou PSTM : Photon Scanning Tunneling Microscopes) atteignent aujourd'hui une résolution latérale importante $(\lambda / 30)$. Cependant, l'expérience et la théorie ont démontré que les images obtenues sont souvent très différentes des profils étudiés. Dans ce travail, nous étendons un modèle précédemment développé pour des configurations bi-dimensionnelles à des configurations tridimensionnelles. Ce modèle est basé sur la résolution des équations de Maxwell avec conditions aux limites au niveau des dioptres composant le dispositif. Nous appliquons ce modèle pour illustrer le fait que le STOM peut détecter les défauts d'indice. Nous montrons qu'il est possible de distinguer des défauts d'indice des défauts de structure du profil. Trois paramètres expérimentaux peuvent influer : la distance de détection, les angles d'incidence et de polarisation.
\end{abstract}

\begin{abstract}
Scanning Tunneling Optical Microscopes (STOM) or Photon Scanning Tunneling Microscopes (PSTM) have succeed to image solid surfaces with high lateral resolution. However, experimental and theoretical studies have proved that images can be very different versus the actual sample profile. In this paper, we extend a previous model based on a perturbative resolution of Maxwell equations, with boundary conditions on a multilayer sample, to the case of three- dimensional devices. As an illustration, STOM can detect index defects. We show that it is feasible to distinguish index defects near the surface of the sample and geometric defects of the profile. Three experimental parameters must be taken into account: the detection distance, the angle of incidence and the polarization of the light can influence the resolution of the index defect.
\end{abstract}

\section{Introduction.}

Scanning Tunneling Optical Microscopes (STOM) or Photon Scanning Tunneling Microscopes (PSTM) have succeed to image solid surfaces with high lateral resolution [1-3]. However, experimental and theoretical studies have proved that images can be very different from the sample profile [4-6]. In addition, the distribution of the refractive index variation of the sample can be found [7,8]. Experimental conditions enable to separate optical refractive index variations and geometrical defects. But we show that when the tip is scanned very close to the sample (a few 
nanometers) and at a constant distance of the sample mean plane, index and geometrical defects may be mistaken one for the other.

In Scanning Tunneling Optical Microscopy, the sample is illuminated by plane waves in total internal reflection. The light diffracted by the sample is collected by an etched optical fiber and is guided to the detector. Because the tip scans at a few nanometers from the sample surface, a realistic model has to take into account the coupling between the tip and the sample. In a previous work, we proposed a model based on a perturbative resolution of Maxwell equations, with boundary conditions on a multilayered 2D sample [9]. In that study, the tip was one layer. We showed the influence of various parameters: metallization of the sample, size of the tip and configuration. In this paper, we extend the model to 3D sample, to illustrate the fact that STOM can detect index defects of nanometric size. It can be one more reason inducing the difference between the profile and the experimental images in near-field. The aim of this paper is to show that small index defect can be distinguished from geometrical sample defect, even in the case of " $z$ constant" images (the tip is scanned in an $x y$ plane, see Fig. 1). Three experimental parameters are studied: the distance of detection, the direction and the polarization of the incoming light that can influence the resolution of the index defect.

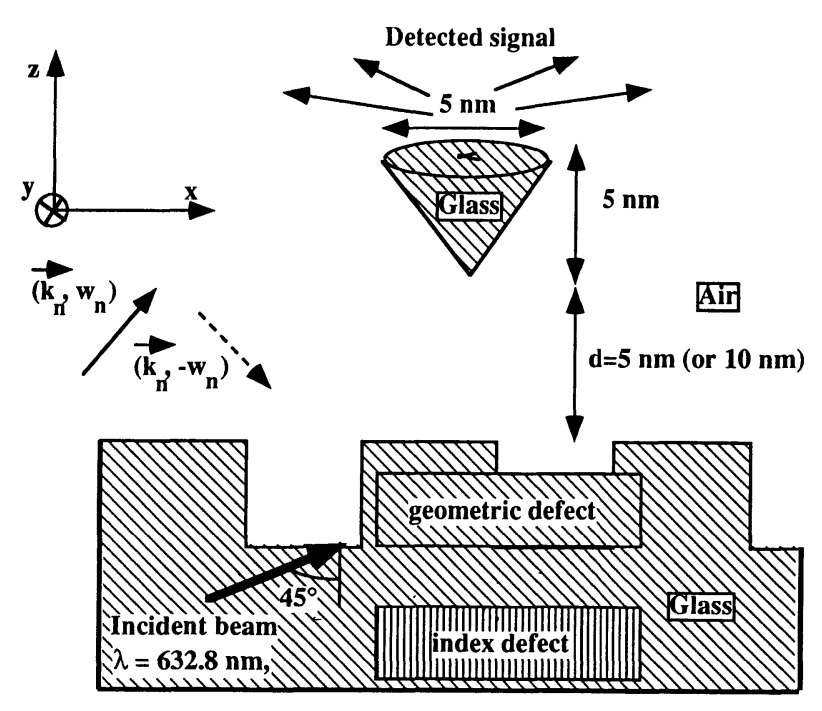

Fig. 1. - Schematic of the multilayer structure used to model Tunneling Optical Microscope principles used in this paper. Illumination is made by transmission, in total internal reflection. Numerical parameters are aforesaid. The glass index is 1.5000 , the defect index is 1.4663 . The grating period is $200 \mathrm{~nm}$, the grating profile is rectangular $(120 \mathrm{~nm}$ wide and $5 \mathrm{~nm}$ high). The defect is a parallelepiped $(210 \mathrm{~nm}$ wide, $210 \mathrm{~nm}$ in length, $3 \mathrm{~nm}$ high). The index defect lays $5 \mathrm{~nm}$ below the grating bottom. The geometrical defect (almost) fills in the gap between two grating tracks.

\section{The model.}

Index defects have been studied in the case of two-dimensional problem in near-field [10]; Pincemin et al. have used a volume integral approach based on Green's dyadic. The case of image enhancement and plasmon resonant effect have been pointed out. In this paper, we use 
the Rayleigh method to study the three-dimensional problem in near-field and we outline angular and distance of detection effects in the non-resonant case. The background of this model is well discribed in our previous papers [6,9]. It is a macroscopic, perturbative, rough multilayer theory based on the resolution of Maxwell equations with boundary conditions (Rayleigh method [11]) The validity of this method is fully discussed in [12] and references therein. The convergence has been verified in our case with regard to the criterion given in [13]. The profile is described by its spatial Fourier harmonics and the ratio of the profile height to the sample dimension (period of the grating) is lower than 0.025 . Moreover, we use $64 * 64$ Fourier harmonics for the calculus and we verified the stability of results by doubling the number of harmonics. In the case of two layers STOM apparatus, we compared our intensity profiles with thus obtained from the grating rigourous differential method in [14]. There is a splendid correlation between the numerical results when the tip sample distance is greater than $2 \mathrm{~nm}$, in the conditions of validity.

The approach takes into account the coupling between the tip and the sample because the tip is one layer of the multilayer device. In this study, the tip is modeled by a small glass cone that is scanned at a few nanometers of the sample. The size of this cone is small with regard to the wavelength and to the period of the sample. Therefore, the usually strong effect of the coupling between the glass tip and the glass sample could be neglected [6]. The main point is a near-field effect and the tip acts like a "passive" dipole.

The diffracted electromagnetic field is analytically calculated and is linearly related to the incident field. This perturbative approach takes advantage in numerical implementation owing to the time consuming and small memory constraint. We can calculate 3D intensity profiles of a 6 layers apparatus in a few minutes with an Apollo HP9000. Nevertheless, this model gives accurate results only if roughness is small compared to the sample lateral size and to the wavelength (in this work, the ratio are lower than $2 \%$ and 5\% resp.). In near field, a ratio of $10 \%$ give the same results as rigourous method. This formalism can be applied to all SNOM (Scanning Near Field Microscopy) devices. The lay-out in figure 1 shows the parameters used for this study.

In previous papers $[6,9]$ we outlined the background of the model. In this one, we extend the model to 3D apparatus and we spread out the characteristics that are usefull in order to understand the way to distinguish index defects.

The Rayleigh expansion of the electromagnetic filed can be expressed as a sum of up and down fields, like in equation (1) (with the assumption of a non periodical multilayer).

$$
\begin{aligned}
\mathbf{E}_{n}(x, y, z) & =\iint_{-\infty}^{+\infty} \mathbf{E}_{n} \uparrow\left(\mathbf{k}_{n}\right) \exp \left[i \mathbf{k}_{n} \cdot \mathbf{r}+i w_{n} z\right] \mathbf{d} \mathbf{k}_{n} \\
& +\iint_{-\infty}^{+\infty} \mathbf{E}_{n} \downarrow\left(\mathbf{k}_{n}\right) \exp \left[i \mathbf{k}_{n} \cdot \mathbf{r}-i w_{n} z\right] \mathbf{d} \mathbf{k}_{n}
\end{aligned}
$$

Where $(\mathbf{r}, z)=(x, y, z)$ are the cartesian coordinates of the point where the field is evaluated and $\left(\mathbf{k}_{n}, w_{n}\right)$ are the coordinates of the wave vector in medium $n,\left(\mathbf{k}_{n}=(u, v)\right)$ and $i=\exp (i \pi / 2)$. The integral is over the diffracted wave vectors in medium $n$ (Plane Wave Spectrum).

If the sample is periodic, the integral becomes a discrete sum [9]. In the case of non absorbing media, diffracted wave vectors can be pure homogeneous (zero imaginary part of $w_{n}$ ) or pure evanescent (zero real part of $w_{n}$ ). If detection is done in far-field, evanescent waves in the detection medium can be neglected. Unlike in near-field, evanescent waves have an important contribution because they are frustrated by the tip and then can be guided by the fiber to the dectector. On the other hand, the higher harmonic is, the greater $\left|w_{n}\right|$ is. Then high spatial frequencies of the sample profile (that are evanescent) are exponentially attenuated. Consequently, the resolution of localized index defects depends obviously on the distance between the tip and the sample. The deeper the index defect lays, the more swiftly it disappears in the detected intensity map, with increasing tip-sample distance. In fact, changing the tip-sample distance can help to characterize index flaws. 
The perturbative calculus (limited to the first two terms), leads to a linear relation between the fields in medium $n-1$ and in medium $n$, related to the Fourier harmonic of the profile $\tilde{h}_{n}\left(\mathbf{k}_{n}\right)$ :

$$
\begin{aligned}
& {\left[\begin{array}{l}
\mathbf{E}_{n} \uparrow\left(\mathbf{k}_{n}\right) \\
\mathbf{E}_{n} \downarrow\left(\mathbf{k}_{n}\right)
\end{array}\right]=\mathbf{M}_{n}\left[\begin{array}{l}
\mathbf{E}_{n-1} \uparrow\left(\mathbf{k}_{n}\right) \\
\mathbf{E}_{n-1} \downarrow\left(\mathbf{k}_{n}\right)
\end{array}\right]+} \\
& \tilde{h}_{n}\left(\mathbf{k}_{n}\right)\left(\varepsilon_{n}-\varepsilon_{n-1}\right) \mathbf{V}_{n} \cdot\left[\begin{array}{l}
\mathbf{E}_{n-1} \uparrow\left(\mathbf{k}_{0}\right) \\
\mathbf{E}_{n-1} \downarrow\left(\mathbf{k}_{0}\right)
\end{array}\right]\left(1-\delta\left(\mathbf{k}_{n}-\mathbf{k}_{0}\right)\right)
\end{aligned}
$$

where $\varepsilon_{n}$ is the permittivity of the medium $n$ and $\delta\left(\mathbf{k}_{n}-\mathbf{k}_{0}\right)$ is the Kronecker symbol. $\left(\mathbf{k}_{0}, w_{0}\right)$ is the incident wave-vector $\left(\mathbf{k}_{0}=\left(u_{0}, v_{0}\right)\right)$. The field in any media can be calculated with equation (2). $\mathbf{M}_{n}$ and $\mathbf{V}_{n}$ are function of $\mathbf{k}_{n}$ and $w_{n}$ or/and $\mathbf{k}_{0}$ and $w_{0}$. Therefore, the angular illumination parameters have to be studied with regards to these matrices.

\section{Results.}

The numerical data are indicated in figure 1. To fit with STOM experimental device, the tip is modeled by a small conical peace of glass. The tip is scanned at a distance $d$ from the top of the grating (5 $\mathrm{nm}$ in Figs. 2-5, and $10 \mathrm{~nm}$ in Figs. 6-7). The sample is a glass dielectric grating (index 1.5). We introduce either a geometrical defect or an optical index defect of a same size: this is a 3 $\mathrm{nm}$ high parallelepiped of glass. Its lateral size is $210 \mathrm{~nm}$. Then this geometrical defect fills in the gap between two gratings grooves. Otherwise, the index defect is a parallelepiped of index 1.4663, $5 \mathrm{~nm}$ under the grating surface. Intensity maps are obtained by calculating the square modulus of the electric field calculated $1 \mathrm{~nm}$ above the tip.

We choose a defect on a rectangular grating to explain more clearly the polarization effects. The grating is an infinite juxtaposition of rectangular track that are parallel to the $y$ axis. On the other hand, the defect is only one parallelepiped. It is limited in the $y$ direction. Then it is easy to compare and to characterize the outcomes of angular filtering in the two directions $x$ and $y$.

We choose a geometrical defect of index 1.5 and an index defect (1.4663) because it is more related to experiment. We can consider that the geometrical flaw is a mistake in making of the grating and that the index defect is a little glass inhomogeneity $(2.3 \%)$. Then the last term in equation (2) is proportional to 0.78 for the optical index defect and to -0.5 for the geometrical defect. Therefore, the resolution of the index defect is not only related to the distance between the defect and the point of detection. Nevertheless, $\exp \left[+i w_{n} z\right]$ acts like a low pass filtering. The diffracted high spatial frequencies are exponentially attenuated for a given distance between tip and the sample. And this effect is stronger than the difference between the two dielectric constants, because the low pass filtering depends exponentionally on the spatial frequencies.

Images are simulated with 1024 gray levels. All levels are used. It is more realistic than absolute intensity because experimental set-up have often such an acquisition apparatus. Nevertheless, we can compare the relative significance of the defect in the image of the grating. Another study could show the absolute intensity level in images, related to the experimental parameters.

In intensity maps, the lower curve is the intensity profile at the bottom of the map. The middle curve is the intensity profile at the middle of the map (defect zone) and the upper curve is the grating profile. Contours show the gratings grooves sides and the defect place. We have studied the result of variations of polarization, direction of incidence and distance of detection

3.1 POLARIZATION AND DIRECTION OF INCIDENCE. - In figures 2 and 3 , the plane of incidence is upright to the grooves, only the polarization differ. In figures 4 and 5 , the plane of incidence is parallel to the grooves. The resolution of the grating seems to be better if a component of the 


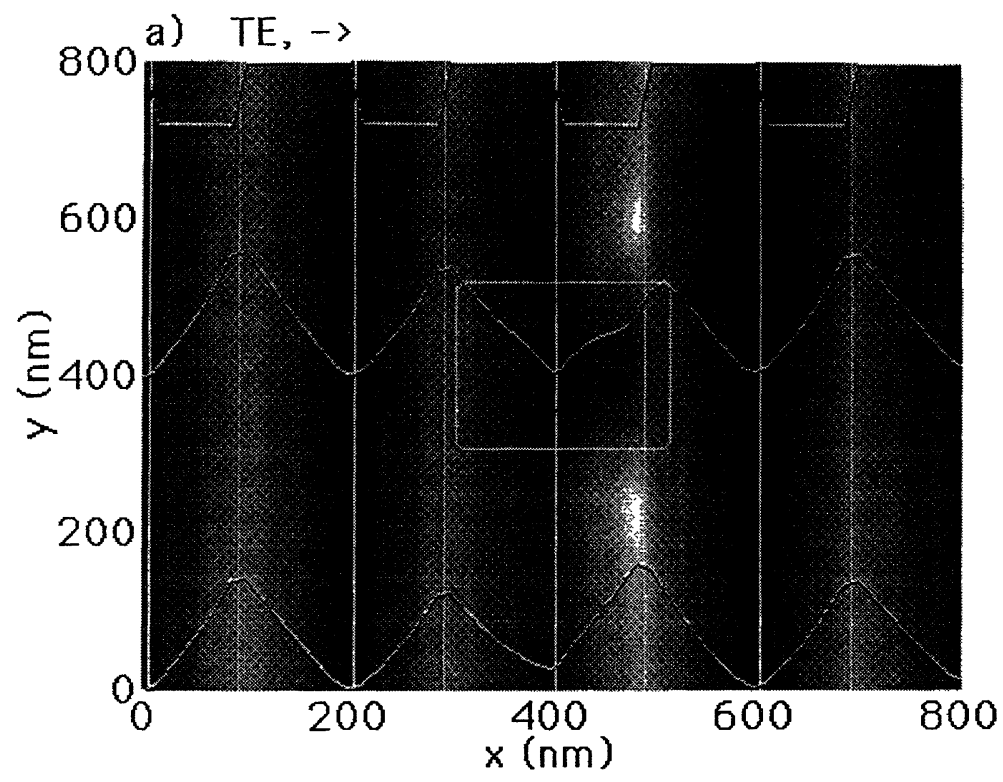

b)

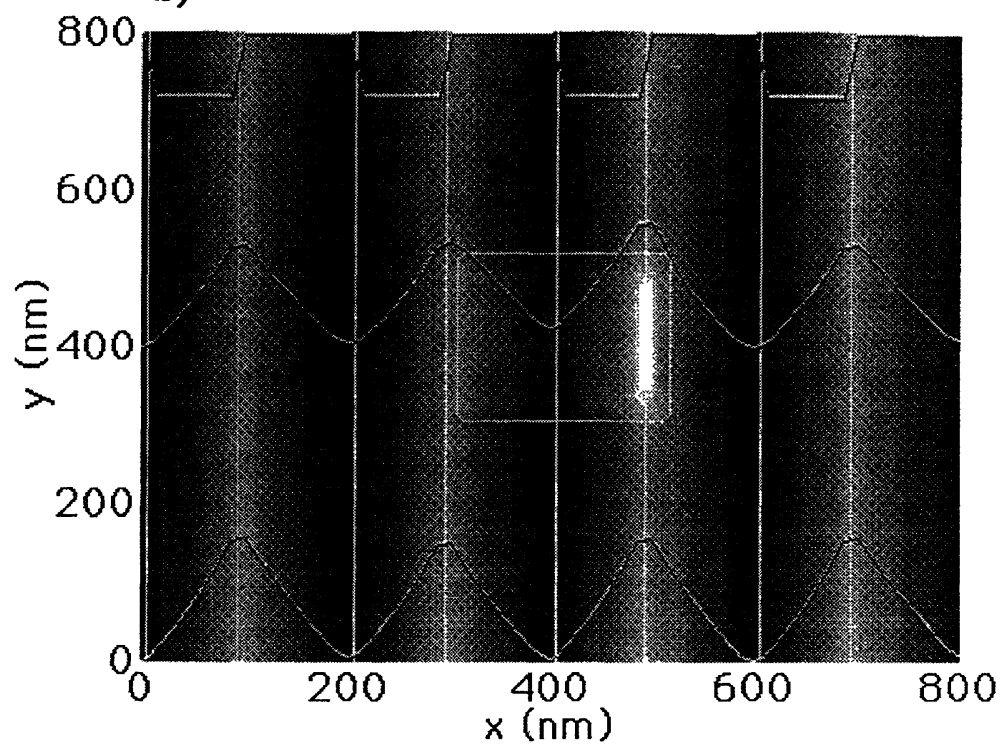

Fig. 2. - Intensity map in grey-level. Numerical parameters are aforesaid in figure 1 . The polarization is $\mathrm{TE}$ and the plane of incidence is upright to the grooves. Incident light comes from the left to the right. The figure a) is calculated for the geometric defect and the figure b) is calculated for the index defect. $d=5 \mathrm{~nm}$.

electric field is upright to the sample profile, because the polarization filtering in $\mathbf{E}_{n} \uparrow\left(\mathbf{k}_{n}\right)$ and in $\mathbf{E}_{n} \downarrow\left(\mathbf{k}_{n}\right)$ more enhances the sample high harmonics in upright direction than in parallel. This first conclusion on polarization effects can be illustrated by comparison of figures 4 and 5 too. But this effect distorts images. In figure 4 (Electric field upright to the grooves), the two edges of the grooves have the main contribution in images. It could leads to a wrong conclusion on the grating profile. In figures 4 and 5, symmetric images illustrate the symmetry of the apparatus. The resolution depends strongly of the electric field direction. For example, the electric field $x$ component 

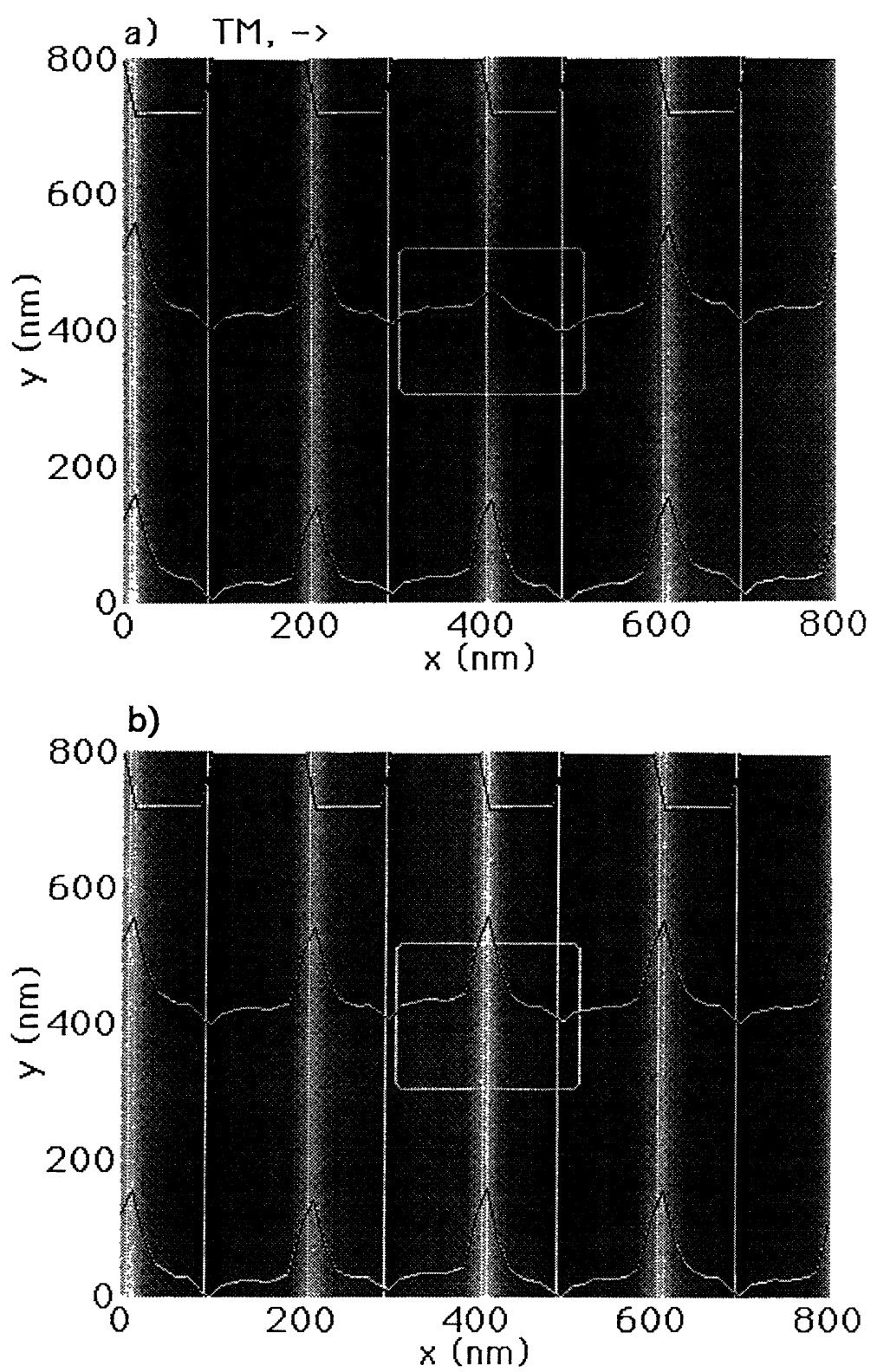

Fig. 3. - Intensity map in grey-level. Numerical parameters are aforesaid in figure 1 . The polarization is $\mathrm{TM}$ and the plane of incidence is upright to the grooves. Incident light comes from the left to the right. The figure a) is calculated for the geometric defect and the figure b) is calculated for the index defect. $d=5 \mathrm{~nm}$.

leads to good resolution in the $y$ direction. This is a well known results in electromagnetic theory. Figures 1 and 3, and figures 2 and 4 make this result clear: one edge of the index defect can appear clearly in the image whenever the grating profile is not well resolved. The electric field $z$ component (if exists) produces another filtering effect that modifies the image contrast (dynamic); but obviously, this effect cannot be observed in 1024 levels images without experimental noise [14, 15]. 

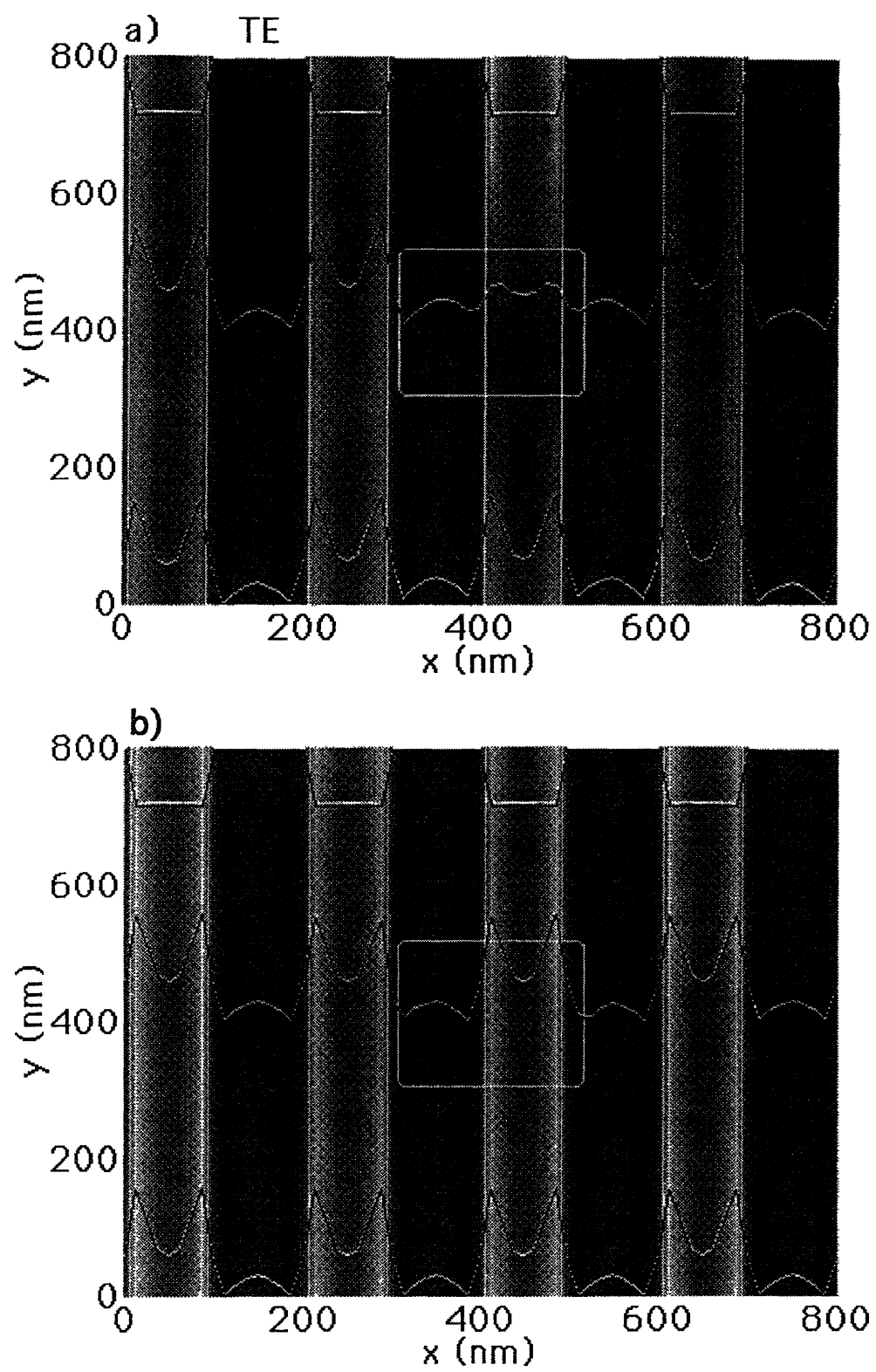

Fig. 4. - Intensity map in grey-level. Numerical parameters are aforesaid in figures 1 and 2. The polarization: TE and the plane of incidence is parallel to the grooves. Incident light comes from the top to the bottom. The figure a) is calculated for the geometric defect and the figure b) is calculated for the index defect. $d=5 \mathrm{~nm}$.

Nevertheless, another strong effect of polarization can be observed: the contrast reversal. In figure 2 the geometrical defect remains in images with constrast reversal. In the same polarization (TE) and for a sample upright to the electric field too, the same effect can be observed for the gratings tracks in figures 4 and 6 . Even if the resolution seems to be better for a detail upright to the electric field, the contrast reversal may conduct to worse conclusion on the sample site. If the incident light direction is parallel to the grooves, in TE mode, the contributions of negative and 

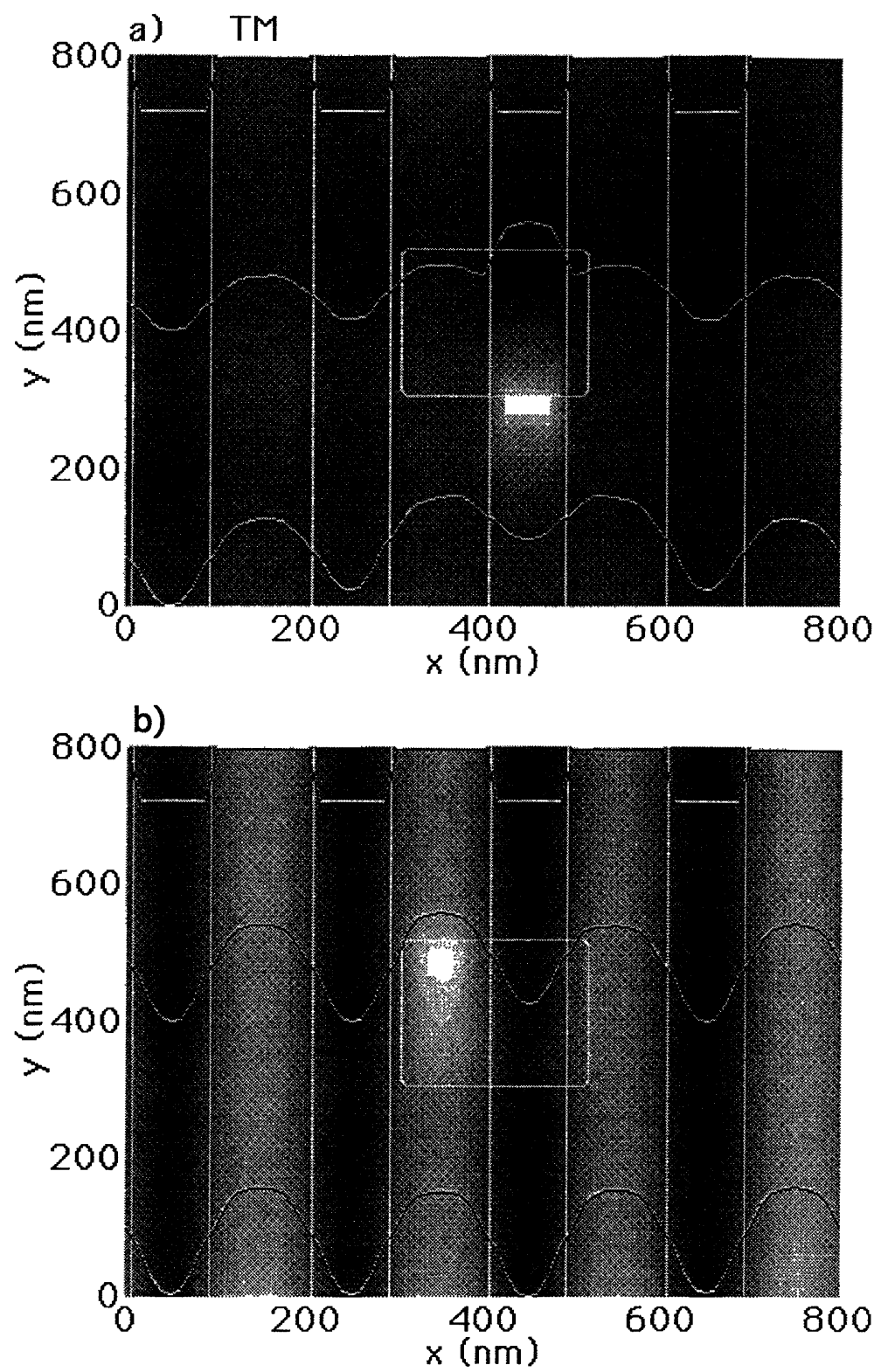

Fig. 5. - Intensity map in grey-level. Numerical parameters are aforesaid in figures 1 and 2. The polarization: TM and the plane of incidence is parallel to the grooves. Incident light comes from the top to the bottom. The figure a) is calculated for the geometric defect and the figure b) is calculated for the index defect. $d=5 \mathrm{~nm}$.

positives harmonics are additives whereas in TM mode they are substractives. If the distance of detection is small, the TE images are the result of destructive interferences of waves associated to the profile spectrum. This is one of the reason of the contrast reversal and of superresolution of the grooves vertical edges in TE mode (Figs. 4 compared to 5). This phenomenon can be observed in figures 2 and 3 in regard to the contrast of the first edge of the grating.

Finally, figures 2 and 3 show dissymetric images: only one edge of the rectangular grating is 

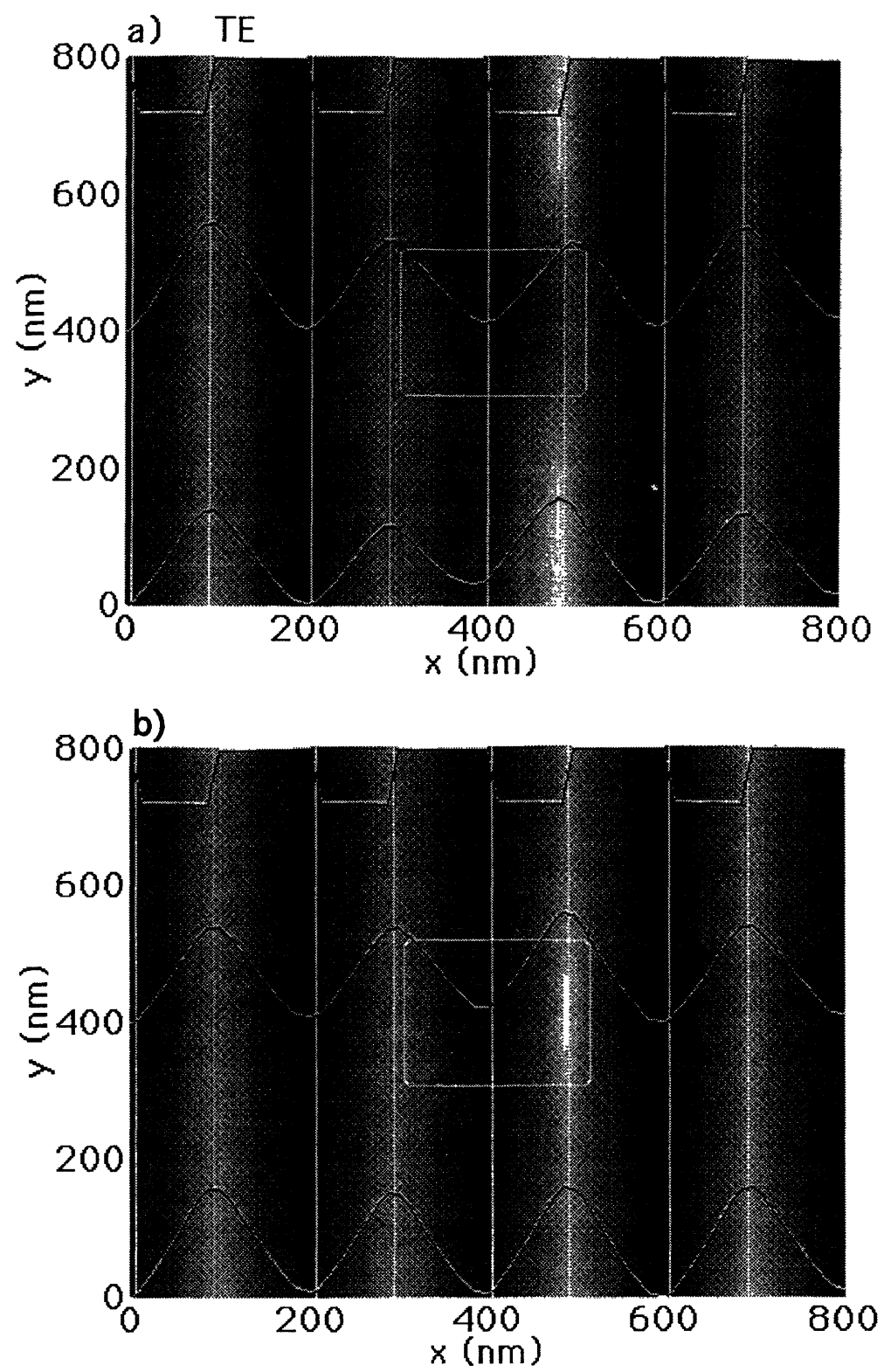

Fig. 6. - Intensity map in grey-level. Numerical parameters are aforesaid in figures 1 and 2 . The polarization: TE and the plane of incidence is upright to the groove. Incident light comes from the left to the right. The figure a) is calculated for the geometric defect and the figure b) is calculated for the index defect. $d=10$ nm.

"resolved". This strong effect can be related to the field confinement [15]. The simplest way to know what edge is resolved is to consider the direction of the incident light direction. In the case of small roughness, the first edge coresponds to a positive derivative and the second to a negative one. Only the first edge of the grating is resolved in figure 2 (TE mode). Only the second edge of the defect is resolved in figure 3 (TM mode) because of the contrast reversal. This effect can only be observed for sample size lower than wavelength and small roughness. 

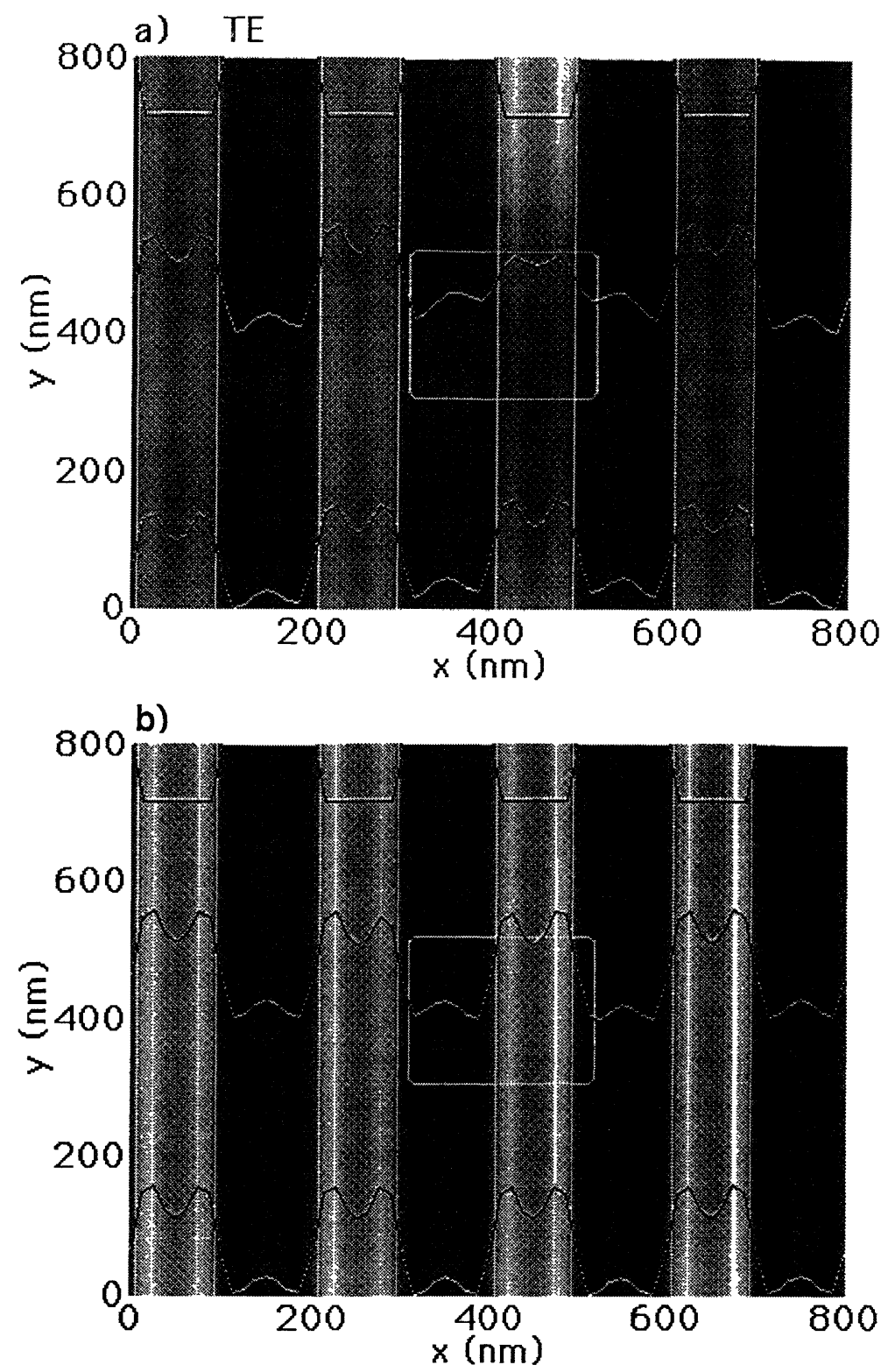

Fig. 7. - Intensity map in grey-level. Numerical parameters are aforesaid in figures 1 and 4 . The polarization: TE and the plane of incidence is parallel to the groove. Incident light comes from the top to the bottom. The figure a) is calculated for the geometric defect and the figure $\mathrm{b}$ ) is calculated for the index defect. $d=10$ $\mathrm{nm}$.

It is important to note that the more resolved the grating is, the less resolved the defect is. Then we can conclude that the main parameter for the resolution is the ratio of the low-pass "propagation" filtering to the high-pass "polarization" filtering.

3.2 DisTANCE OF DETECTION EFFECT. - If we compare figures 2 and 4 to figures 6 and 7, the influence of the distance of detection clearly appears. The deeper the defect is, the worse resolved 
it is. The high frequency of the sample are attenuated with the distance, this phenomenon is very clear in figure 7 , by comparison with figure 4 . This strong effect can be observed by looking at the defect survival in images. The consequence of this effect is that the tip has to be very close to the sample, to detect information on small details. It appears numerically that the resolution criteria defined in [16] can be extended to any non-periodic sample: for a detail with a lateral size of $\lambda / 3$ $(210 \mathrm{~nm})$, the distance of detection must be lower than $10 \mathrm{~nm}$. This result and the polarization effects point out a tremendous problem in near-field microscopies: what is the meaning of resolution? Is it only the survival of the dim position of a detail or is it the resolution of the detail shape? This study gives illustation of this question. A near-field image can show one side of a detail, the detail position or the detail geometry. The intensity profile is ever distorted compared to the real profile: two filtering effects act in the opposite way: the "distance" low-pass that ruins, and "angular" high-pass that enhances too much. Then it is difficult to have "good" images in STOM because STOM "see" more than the profile.

\section{Conclusion.}

In this paper we have extended a previous model to three-dimensional STOM device. In particular, we have presented results related to the resolution of an index defect that lays under the sample surface. We chosed the same optical index for the geometrical defect as for the grating to consider a pure geometrical variation. We show that constant distance images can contain information on the optical index defect. Moreover, increasing the distance of detection enables to release the image of small defects. Moreover, having a good knowledge of the defects size can help to choose the suitable lightening to discriminate index defects. Then, separating information on the index and on the geometry remains possible. The index defect has a complete influence on images because it appears like a phase defect. But it lays under the surface profile, then its contribution is reduce by the "distance" filtering. On the opposite, the geometric defect has a dissymetric contribution in images because it fills almost in the gap between two tracks, and its contribution remains more important when increasing the tip-sample distance despite a given lateral size. Then the way to distinguish the two defects is multi-acquisition (polarization and distance).

\section{References}

[1] Pohl D., "Scanning near-field optical microscopy" in Advances in Optical and Electron Microscopy, C.R.J. Sheppard and T. Mulvey Eds. (Academic Press, Londre, 1990).

[2] Courjon D. , Bainier C. , Spajer M. , "Imaging of submicron index variations by scanning optical tunneling", J. Vac. Sci. Technol. B10 (1992) 2436.

[3] Near Field Optics, D. Pohl and D. Courjon Eds. (Kluwer Acad., 1993).

[4] Moers M.H.P., Tack R.G., Noordman O.F.J., Segerink F.B., Van Hulst N.F., Bölger B., "Combined photon scanning tunneling microscope and atomic force using silicon nitride probes", Near Field Optics, D. Pohl and D. Courjon Eds. (Kluwer Acad., 1993) 79-86.

[5] Nevière M., Vincent P., "Diffraction gratings as components for photon scanning tunneling microscope image interpretation", Near Field Optics, D. Pohl and D. Courjon Eds. (Kluwer Acad., 1993) 377378.

[6] Van Labeke D., Barchiesi D., "Probes for scanning tunneling optical microscopy: a theoretical comparison”, J. Opt. Soc. Am. A10 (1993) 2193-2201. 
[7] De Fornel F., Bourillot E., Adam P., Salomon L., Chabrier G., Goudonnet J.P., "Recent experimental results with the PSTM: - observation of a step on a quartz surface. - spatial spectroscopy of microwaveguides", Near Field Optics, D. Pohl and D. Courjon Eds. (Kluwer Acad., 1993) 59-70.

[8] Courjon D., Bainier C., Spajer M., "Imaging of submicron index variations by scanning optical tunneling", J. Vac. Sci. Technol. B10 (1992) 1-3.

[9] Barchiesi D., Van Labeke D., "Une modélisation de la métallisation des objets et de la forme des sondes", Microsc. Microanal. Microstruct. 5 (1994) 1-10.

[10] Pincemin F., Santenac A., Greffet J.J., "Near field scattered by a dielectric rod below a metallic surface", J. Opt. Soc. Am. A11 (1994) 117-1127.

[11] Toigo F. Marvin A., Celli V., Hill N.R., "Optical properties of rough dielectric surfaces: general theory and the small roughness limit", Phys. Rev. B15 (1978) 5618-5626.

[12] Petit R., Electromagnetic Theory of gratings , Vol. 22 of Topics in Current Physics (Springer Verlag, 1980).

[13] Van den Berg P.M., Fokkema J.T., "The Rayleigh hypothesis in the theory of reflection by gratings”, J. Opt. Soc. Am. 69 (1979) 27-31.

[14] Barchiesi D., "Modélisation des microscopes optiques en champ proche STOM et SNOM. Optimisation et Spectroscopie" PhD Thesis, Sect. III.3.a and Sect. III.3.b (Université de Franche-Comté, France, 1993).

[15] Girard C., Dereux A., Martin O.J.F., “Theory of Near Field Optics", To be published in NATO "Photon and Local Probe" (1995).

[16] Van Labeke D., Barchiesi D., "Scanning-tunneling optical microscopy: a theoretical macroscopic approach", J. Opt. Soc. Am. A9 (1992) 732-739. 